\title{
A generalization of Maillet and Demyanenko determinants
}

\author{
by \\ Mikinito Hirabayashi (Ishikawa)
}

1. Introduction. Let $K$ be an imaginary abelian number field with conductor $m$ and of degree $[K: \mathbb{Q}]=2 n$. Let $Q_{K}$ be the unit index of $K$ and $w_{K}$ the number of roots of unity in $K$. Then the relative class number $h_{K}^{*}$ of $K$ is given by

$$
h_{K}^{*}=Q_{K} w_{K} \prod_{\chi} \frac{1}{2 f(\chi)} \sum_{a=1}^{f(\chi)}(-\chi(a) a)
$$

where the product $\prod_{\chi}$ is taken over the odd primitive characters $\chi$ of $K$ with conductor $f(\chi)$ (see Hasse [4]).

Several ways of representing the product in (1) by a determinant are known, some of them holding for certain types of fields only (see Carlitz and Olson [1], Hazama [5] and further references in Hirabayashi [6]). Here our main concern is a unified approach to the Maillet determinant on the one hand and to the Demyanenko determinant on the other hand. This approach relies on the " $b$-division vector" introduced by Girstmair [3]. We obtain a relative class number formula for an arbitrary imaginary abelian number field which generalizes formulae of Girstmair [2] and [6]. Tsumura [7] also generalized both type of determinant formulae, but his generalization for the Demyanenko determinant requires the oddness of the conductor.

2. Lemma obtained by the $b$-division vector. In this section we state one of the results of [3] which we need to describe our results.

For $m \in \mathbb{Z}, m \geq 3, m \not \equiv 2 \bmod 4$ let $G_{m}=(\mathbb{Z} / m \mathbb{Z})^{\times}$be the prime residue group $\bmod m$ and $H$ a subgroup of $G_{m}$ of relative index $q=\left[G_{m}\right.$ : $H]=\left|G_{m} / H\right|$. We assume that $H$ does not contain the class $\overline{-1}$, letting $\bar{x}=x+m \mathbb{Z}$ for $x \in \mathbb{Z}$.

1991 Mathematics Subject Classification: Primary 11R29; Secondary 11R20. 
For a class $C \in G_{m} / H$ and for an integer $b, b \geq 2, m \nmid b$, let

$$
S_{C}^{(b)}=\sum_{\bar{k} \in C, 0<k<m}\left[\frac{b k}{m}\right],
$$

where $[x]$ denotes the integral part of a real number $x$, and let

$$
S^{(b)}=\frac{1}{q} \sum_{C \in G_{m} / H} S_{C}^{(b)} \quad \text { and } \quad T_{C}^{(b)}=S_{C}^{(b)}-S^{(b)} .
$$

Then we have

$$
T_{-C}^{(b)}=-T_{C}^{(b)},
$$

where $-C=\left\{\overline{-x} \in G_{m}: \bar{x} \in C\right\}$.

Since $H$ does not contain $\overline{-1}$, letting $n=q / 2$, we can take classes $C_{1}, C_{2}, \ldots, C_{n}$ of $G_{m} / H$ which satisfy

$$
G_{m} / H=\left\{C_{1},-C_{1}, C_{2},-C_{2}, \ldots, C_{n},-C_{n}\right\} .
$$

Then $R=\left\{C_{1}, \ldots, C_{n}\right\}$ can be regarded as a complete system of representatives for $G_{m} /(H\{\overline{ \pm 1}\})$. We assume $R \ni H$.

We call the vector $T^{(b)}=\left(T_{C}^{(b)}\right)_{C \in R}$ the b-division vector of $G_{m} / H$. (For an explanation of this name, see [3].)

When a Dirichlet character $\chi$ is defined $\bmod m$, we sometimes use $\chi_{m}$ instead of $\chi$. We denote by $\chi_{f}$ the primitive character corresponding to $\chi$.

For a Dirichlet character $\chi=\chi_{m}$ let

$$
B_{\chi}=B_{\chi_{m}}=\frac{1}{m} \sum_{\substack{k=1 \\ k, m)=1}}^{m} \chi(k) k .
$$

Then we have

$$
B_{\chi_{m}}=\prod_{p \mid m}\left(1-\chi_{f}(p)\right) \cdot B_{\chi_{f}},
$$

where the product $\prod_{p \mid m}$ is taken over the primes $p$ dividing $m$.

For integers $m$ and $b$ we let $m_{b}=(m, b)$ be the greatest common divisor of $m$ and $b$, and let $m^{\prime}=m / m_{b}, b^{\prime}=b / m_{b}$.

Lemma $1([3,(17)-(20)])$. Let the notation be as above. Then, for an odd character $\chi=\chi_{m}$, we have

$$
\frac{1}{2} c_{\chi}(b) B_{\bar{\chi}_{f}}=\sum_{C \in R} \bar{\chi}(C) T_{C}^{(b)}
$$

where $\bar{\chi}$ is the complex conjugate character of $\chi$, i.e., $\bar{\chi}(C)=\overline{\chi(C)}$ and 
$c_{\chi}(b)$ is defined by

$$
c_{\chi}(b)= \begin{cases}b \prod_{p \mid m}\left(1-\bar{\chi}_{f}(p)\right) & \text { if } f(\chi) \nmid m^{\prime}, \\ m_{b} \prod_{p \mid m^{\prime}}\left(1-\bar{\chi}_{f}(p)\right) \cdot c^{\prime} & \text { if } f(\chi) \mid m^{\prime}\end{cases}
$$

with

$$
c^{\prime}=b^{\prime} \prod_{p \mid m, p \nmid m^{\prime}}\left(1-\bar{\chi}_{f}(p)\right)-\chi_{m^{\prime}}\left(b^{\prime}\right) \prod_{p \mid m, p \nmid m^{\prime}}\left(1-\frac{1}{p}\right),
$$

$\chi_{m^{\prime}}$ being the Dirichlet character mod $m^{\prime}$ belonging to $\chi$.

As usual, we let $\prod_{p \mid m, p \nmid m^{\prime}}$ be equal to 1 if there is no prime $p$ such that $p \mid m$ and $p \nmid m^{\prime}$.

3. Results. Let $K$ be an imaginary abelian number field with conductor $m$. Let $H$ be the subgroup of $G_{m}=(\mathbb{Z} / m \mathbb{Z})^{\times}$corresponding to $K$. Let $X^{-}$ be the set of odd characters defined $\bmod m$ of $K$. Put

$$
c_{K}^{*}(b)=\prod_{\chi \in X^{-}} c_{\chi}(b)
$$

TheOREM. Let $K$ be an imaginary abelian number field with conductor $m$ and of degree $2 n$. Let $b \geq 2$ be an integer not divisible by $m$. Then

$$
\begin{aligned}
c_{K}^{*}(b) h_{K}^{*} & =(-1)^{n} Q_{K} w_{K} \operatorname{det}\left(T_{C_{1} C_{2}^{-1}}^{(b)}\right)_{C_{1}, C_{2} \in R} \\
& = \pm Q_{K} w_{K} \operatorname{det}\left(T_{C_{1} C_{2}}^{(b)}\right)_{C_{1}, C_{2} \in R} .
\end{aligned}
$$

When $b=m+1$ and $b=2$, we have the following Corollary, where the determinants $\operatorname{det}\left(T_{C_{1} C_{2}^{-1}}^{(b)}\right)_{C_{1}, C_{2} \in R}$ turn out to be the Maillet determinant and the Demyanenko determinant, respectively.

For $c \in \mathbb{Z},(c, m)=1$ we let $c^{-1}$ be an integer such that $c c^{-1} \equiv 1 \bmod m$ and let

$$
C(c)=|\bar{c} H \cap\{a \bmod m: 1 \leq a<m / 2\}| .
$$

Let

$$
g_{K}^{*}=\prod_{\chi \in X^{-}} \prod_{p \mid m}\left(1-\chi_{f}(p)\right), \quad F_{K}=\prod_{\chi \in X^{-}}\left(2-\chi_{f}(2)\right)
$$

and

$$
e_{K}= \begin{cases}1 & \text { if } m \text { is odd, or if } m \text { is even and } \chi((m-2) / 2)=+1 \text { for every } \\ & \chi \in X^{-} \\ 0 & \text { otherwise. }\end{cases}
$$

COROLlary. Let $K$ be an imaginary abelian number field with conductor $m$ and of degree $2 n$. Then we have the following formulae of [2] and [6], 
respectively:

(5) $g_{K}^{*} h_{K}^{*}=(-1)^{n} \frac{Q_{K} w_{K}}{m^{n}} \operatorname{det}\left(\sum_{\bar{k} \in C_{1} C_{2}^{-1}, 0<k<m}\left(k-\frac{m}{2}\right)\right)_{C_{1}, C_{2} \in R}$,

$$
e_{K} g_{K}^{*} h_{K}^{*}=(-1)^{n} \frac{Q_{K} w_{K}}{F_{K}} \operatorname{det}\left(\frac{\varphi(m)}{2 q}-C\left(c_{1} c_{2}^{-1}\right)\right)_{C_{1}, C_{2} \in R}
$$

where $\varphi$ is Euler's totient function and $C_{i}=\bar{c}_{i} H$ for $i=1,2$.

\section{Proofs of Theorem and Corollary}

Proof of Theorem. By the formula (1) and Lemma 1 we have

$$
\begin{aligned}
c_{K}^{*}(b) h_{K}^{*} & =Q_{K} w_{K} \prod_{\chi \in X^{-}}\left(-\frac{1}{2} c_{\chi}(b) B_{\bar{\chi}_{f}}\right) \\
& =(-1)^{n} Q_{K} w_{K} \prod_{\chi \in X^{-}} \sum_{C \in R} \bar{\chi}(C) T_{C}^{(b)} .
\end{aligned}
$$

Here we fix one odd character $\chi_{1}$ of $K$. Then

$$
\begin{aligned}
\prod_{\chi \in X^{-}} \sum_{C \in R} \bar{\chi}(C) T_{C}^{(b)} & =\prod_{\chi \in X^{-}} \sum_{C \in R} \chi(C) T_{C}^{(b)} \\
& =\prod_{\chi_{0} \in X^{+}} \sum_{C \in R}\left(\chi_{0}(C) \cdot \chi_{1}(C) T_{C}^{(b)}\right),
\end{aligned}
$$

where $X^{+}$is the group of even characters of $K$.

Noting that $R$ is regarded as a complete system of representatives of the group $G_{m} /(H\{\overline{ \pm 1}\})$ and that $X^{+}$is the group of characters of $G_{m} /(H\{\overline{ \pm 1}\})$, by the group determinant formula (cf. [4, p. 23]) we have

$$
\begin{aligned}
\prod_{\chi \in X^{-}} \sum_{C \in R} \bar{\chi}(C) T_{C}^{(b)} & =\operatorname{det}\left(\chi_{1}\left(C_{1} C_{2}^{-1}\right) T_{C_{1} C_{2}^{-1}}^{(b)}\right)_{C_{1}, C_{2} \in R} \\
& =\operatorname{det}\left(T_{C_{1} C_{2}^{-1}}^{(b)}\right)_{C_{1}, C_{2} \in R} .
\end{aligned}
$$

Since $\left\{C^{-1} \in G_{m} / H: C \in R\right\}$ is also a complete system of representatives of $G_{m} /(H\{\overline{ \pm 1}\})$, by the formula (2) we have

$$
\operatorname{det}\left(T_{C_{1} C_{2}^{-1}}^{(b)}\right)_{C_{1}, C_{2} \in R}= \pm \operatorname{det}\left(T_{C_{1} C_{2}}^{(b)}\right)_{C_{1}, C_{2} \in R} .
$$

Thus we get the desired formulae (3) and (4).

For the proof of the Corollary we need

LEMma 2. Suppose that the conductor $m$ of an imaginary abelian number field $K$ is even. Then, for an odd character $\chi$ of $K, \chi((m-2) / 2)=+1$ if and only if the 2-part of the conductor $f(\chi)$ coincides with that of $m$. 
Proof. If $f(\chi)$ divides $m / 2$, then $\chi((m-2) / 2)=\chi(-1)=-1$.

Conversely, we assume that the 2-part of the conductor $f(\chi)$ coincides with that of $m$. Let the character $\chi$ be decomposed into the characters $\xi_{1}$ and $\xi_{2}$ with 2-power conductor and odd conductor, respectively: $\chi=\xi_{1} \xi_{2}$.

If $4 \| f(\chi)$, then $\xi_{1}((m-2) / 2)=\xi_{2}((m-2) / 2)=+1$ and therefore $\chi((m-2) / 2)=+1$.

If $8 \mid f(\chi)$, then $\xi_{1}((m-2) / 2)=\xi_{2}((m-2) / 2)=-1$ or +1 according as $\xi_{1}(-1)=+1$ or -1 . Hence we have $\chi((m-2) / 2)=+1$.

Proof of Corollary. First we consider the case of $b=m+1$. As shown in [3], we have

Therefore,

$$
S^{(b)}=\frac{m \varphi(m)}{2 q} \quad \text { and } \quad S_{C}^{(m+1)}=\sum_{\bar{k} \in C, 0<k<m} k .
$$

$$
T_{C}^{(m+1)}=S_{C}^{(m+1)}-S^{(m+1)}=\sum_{\bar{k} \in C, 0<k<m}\left(k-\frac{m}{2}\right) .
$$

On the other hand, since $m^{\prime}=m$ and $b^{\prime}=b$, by Lemma 1 we have

$$
c_{\chi}(b)=m_{b} \prod_{p \mid m^{\prime}}\left(1-\bar{\chi}_{f}(p)\right) \cdot c^{\prime}=m \prod_{p \mid m}\left(1-\bar{\chi}_{f}(p)\right) .
$$

Hence $c_{K}^{*}(b)=m^{n} g_{K}^{*}$.

Thus, by the formulae (3) and (7) we obtain

$$
m^{n} g_{K}^{*} h_{K}^{*}=(-1)^{n} Q_{K} w_{K} \operatorname{det}\left(\sum_{\bar{k} \in C_{1} C_{2}^{-1}, 0<k<m}\left(k-\frac{m}{2}\right)\right)_{C_{1}, C_{2} \in R},
$$

which is our desired formula (5).

Secondly we consider the case of $b=2$. By [3], we have

and

$$
T_{C}^{(2)}=S_{C}^{(2)}-\frac{\varphi(m)}{2 q}
$$

$$
S_{C}^{(2)}=|\{\bar{k} \in C: m / 2<k<m\}|=\frac{\varphi(m)}{q}-C(c),
$$

where $C=\bar{c} H$. Consequently, we have

$$
T_{C}^{(2)}=\frac{\varphi(m)}{2 q}-C(c) \quad \text { for } C=\bar{c} H .
$$

Now we let $m$ be odd. Since $m^{\prime}=m, b^{\prime}=2$, it follows from Lemma 1 that

$$
c_{\chi}(b)=\prod_{p \mid m}\left(1-\bar{\chi}_{f}(p)\right) \cdot(2-\chi(2))
$$

and hence $c_{K}^{*}(b)=g_{K}^{*} F_{K}$. 
Thus, by the formulae (3) and (8) we obtain

$$
F_{K} g_{K}^{*} h_{K}^{*}=(-1)^{n} Q_{K} w_{K} \operatorname{det}\left(\frac{\varphi(m)}{2 q}-C\left(c_{1} c_{2}^{-1}\right)\right)_{C_{1}, C_{2} \in R},
$$

where $C_{1}=\bar{c}_{1} H$ and $C_{2}=\bar{c}_{2} H$.

Because of the oddness of $m$ we have $e_{K}=1$. Thus we get the formula (6) under this condition.

Next we let $m$ be even. Then we have $m^{\prime}=m / 2, b^{\prime}=1$.

If for some odd character $\chi$ of $K$ the conductor $f(\chi)$ divides $m^{\prime}$, then $\chi((m-2) / 2)=-1$ and so $e_{K}=0$.

On the other hand, by Lemma 1 we have

$$
c_{\chi}(b)=2 \prod_{p \mid m^{\prime}}\left(1-\bar{\chi}_{f}(p)\right) \cdot c^{\prime}
$$

and $c^{\prime}=0$. Hence, $c_{K}^{*}(b)=0$. Then we see by the Theorem that $\operatorname{det}\left(T_{C_{1} C_{2}^{-1}}^{(2)}\right)$ $=0$. Thus we have proved the formula (6) under this condition.

Finally, we suppose that for every $\chi \in X^{-}$the 2-part of the conductor $f(\chi)$ coincides with that of $m$. Then it follows from Lemma 1 that

$$
c_{\chi}(b)=2 \prod_{p \mid m}\left(1-\bar{\chi}_{f}(p)\right) \quad \text { for every } \chi \in X^{-}
$$

and hence $c_{K}^{*}(b)=2^{n} g_{K}^{*}$. Consequently, from (3) and (8) we obtain

$$
2^{n} g_{K}^{*} h_{K}^{*}=(-1)^{n} Q_{K} w_{K} \operatorname{det}\left(\frac{\varphi(m)}{2 q}-C\left(c_{1} c_{2}^{-1}\right)\right)_{C_{1}, C_{2} \in R},
$$

where $C_{1}=\bar{c}_{1} H$ and $C_{2}=\bar{c}_{2} H$.

By Lemma 2 we have $e_{K}=1$. And under this condition we get $F_{K}=2^{n}$. Thus we obtain the formula (6).

Acknowledgements. I would like to express my sincere thanks to Professor Kurt Girstmair for posing this problem and many valuable suggestions. I would also like to express my sincere thanks to the referee for many valuable suggestions which improved this paper.

\section{References}

[1] L. Carlitz and F. R. Olson, Maillet's determinant, Proc. Amer. Math. Soc. 6 (1955), 265-269.

[2] K. Girstmair, The relative class numbers of imaginary cyclic fields of degrees 4, 6 , 8 and 10, Math. Comp. 61 (1993), 881-887.

[3] - Class number factors and distribution of residues, Abh. Math. Sem. Univ. Hamburg 67 (1997), to appear. 
[4] H. Hasse, Über die Klassenzahl abelscher Zahlkörper, Akademie-Verlag, 1952 (reproduction: Springer, 1985).

[5] F. Hazama, Demjanenko matrix, class number, and Hodge group, J. Number Theory $34(1990), 174-177$.

[6] M. Hirabayashi, A relative class number formula for an imaginary abelian field by means of Demjanenko matrix, in: Proceedings of the Conference on Analytic and Elementary Number Theory (Vienna, July 18-20, 1996), W. G. Nowak and J. Schoißengeier (eds.), Universität Wien and Universität für Bodenkultur, 1997, $81-91$.

[7] H. Tsumura, On Demjanenko's matrix and Maillet's determinant for imaginary abelian number fields, J. Number Theory 60 (1996), 70-79.

Kanazawa Institute of Technology

7-1, Ogigaoka, Nonoichi-machi

Ishikawa 921, Japan

E-mail: hira@neptune.kanazawa-it.ac.jp

Received on 24.3.1997

and in revised form on 18.8.1997 